\title{
Composite (Goldstone) Higgs Dynamics on the Lattice: Spectrum of SU(2) Gauge Theory with two Fundamental Fermions
}

\author{
Rudy Arthur \\ E-mail: rudy.d.arthur@gmail.com

\section{Vincent Drach} \\ E-mail: drachecp3-origins.net

\section{Martin Hansen} \\ E-mail: hansen@cp3-origins. net
}

\section{Ari Hietanen*}

E-mail: hietanen@cp3-origins.net

\section{Randy Lewis}

E-mail: randy. lewis@yorku.ca

\section{Claudio Pica}

E-mail: pica@cp3-origins.net

\section{Francesco Sannino}

E-mail: sannino@cp3-origins.net

We study the meson spectrum of the $\mathrm{SU}(2)$ gauge theory with two Wilson fermions in the fundamental representation. The theory unifies both Technicolor and composite Goldstone Boson Higgs models of electroweak symmetry breaking. We have calculated the masses of the lightest spin one vector and axial vector mesons. In addition, we have also obtained preliminary results for the mass of the lightest scalar (singlet) meson state. The simulations have been done with multiple masses and two different lattice spacings for chiral and continuum extrapolations. The spin one meson masses set lower limits for accelerator experiments, whereas the scalar meson will mix with a pGB of the theory and produce two scalar states. The lighter of the states is the $125 \mathrm{GeV}$ Higgs boson, and the heavier would be a new yet unobserved scalar state.

CP3-Origins-2014-046 DNRF90 \& DIAS-2014-46

The 32nd International Symposium on Lattice Field Theory,

23-28 June, 2014

Columbia University New York, NY

\footnotetext{
* Speaker.
} 


\section{Introduction}

The SU(2) gauge theory with two fundamental Dirac fermions is the simplest field theoretical realization of unified theory of Composite Goldstone Boson Higgs (CGBH) and Technicolor [1]. The theory has a chiral symmetry breaking pattern from $\mathrm{SU}(4) \rightarrow \mathrm{SP}(4) \sim \mathrm{SO}(5)$ which gives five Goldstone Bosons [2,3]. The breaking direction with respect to standard model can be parametrized by an angle $\theta$. There are two extreme cases $\theta=0$ and $\theta=\pi / 2$. The former corresponds to a composite Goldstone Boson Higgs model and the latter to a Technicolor model.

In the CGBH limit the electroweak symmetry is intact, i.e., it resembles the standard model with a zero Higgs mass. The four components of the Higgs doublet are composed of four Goldstone Bosons. The fifth GB is standard model neutral.

In the Technicolor limit the electroweak symmetry is completely broken, with three of the five Goldstones eaten by the SM gauge bosons. The Higgs particle is the lightest scalar $\sigma$ in the SU(2) gauge theory. The remaining GB form a complex doublet which is a possible asymmetric dark matter candidate [3].

The value of $\theta$ is determined dynamically. Besides the electroweak and top induced radiative contributions its final value depends also on new possible operators breaking the original SU(4) global symmetry explicitly. A generic value of $\theta$ between the two extreme cases is therefore naturally expected [1]. In this case the composite GB Higgs state mixes with the Technicolor $\sigma$ state giving two scalar particles of which the lightest one is identified as the Higgs Boson. The scalar masses obtain large corrections from the coupling to top $[4,1]$ whereas the (axial)vector meson masses are mostly determined by the dynamics of the SU(2) gauge theory. The scale of the theory is $f_{\Pi} \sin \theta=246 \mathrm{GeV}$.

Here we will review lattice calculations of the model performed in [5] (see also [7]) and present preliminary results of measuring the mass of the scalar meson. Scattering lengths of the same model has been studied in [6]. In addition to the unified composite Goldstone Boson dynamics the SU(2) theory with two fundamental fermions has been used widely in beyond standard model phenomenology and dark matter studies $[3,8,9,10]$.

\section{Theory}

We use the standard Wilson plaquette action with Wilson fermions.

$$
\begin{aligned}
S_{W}= & \frac{\beta}{2} \sum_{x, \mu, v}\left(1-\frac{1}{2} \operatorname{Re} \operatorname{Tr} U_{\mu}(x) U_{v}(x+\hat{\mu}) U_{\mu}^{\dagger}(x+\hat{v}) U_{v}^{\dagger}(x)\right)+\sum_{x} \bar{\psi}(x)\left(4+m_{0}\right) \psi(x) \\
& -\frac{1}{2} \sum_{x, \mu}\left(\bar{\psi}(x)\left(1-\gamma_{\mu}\right) U_{\mu}(x) \psi(x+\hat{\mu})+\bar{\psi}(x+\hat{\mu})\left(1+\gamma_{\mu}\right) U_{\mu}^{\dagger}(x) \psi(x)\right),
\end{aligned}
$$

where $U_{\mu}$ is the gauge field and $\beta$ the gauge coupling in conventional lattice notation. $\psi$ is the doublet of $u$ and $d$ fermions, and $m_{0}$ is the mass matrix. 
We extract the non singlet meson masses from the two-point correlation functions

$$
\begin{aligned}
C_{\bar{u} d}^{(\Gamma)}\left(t_{i}-t_{f}\right) & =\sum_{\bar{x}_{i}, \vec{x}_{f}}\left\langle\mathscr{O}_{u d}^{(\Gamma)}\left(x_{f}\right) \mathscr{O}_{u d}^{(\Gamma) \dagger}\left(x_{i}\right)\right\rangle \\
& =\sum_{\bar{x}_{i}, \vec{x}_{f}} \operatorname{Tr} \Gamma S_{d \bar{d}}\left(x_{f}, x_{i}\right) \gamma^{0} \Gamma^{\dagger} \gamma^{0} S_{u \bar{u}}\left(x_{i}, x_{f}\right),
\end{aligned}
$$

where $S_{u \bar{u}}(x, y)=\langle u(x) \bar{u}(y)\rangle$. The quantities of interest are pseudoscalar $\Gamma=\gamma_{5}$, vector $\Gamma=\gamma_{k}$ $(k=1,2,3)$, and axial vector $\Gamma=\gamma_{5} \gamma_{k}$ mesons. We use stochastic estimator for the non singlet correlators with $Z_{2} \times Z_{2}$ single time slice stochastic sources.

The correlator for scalar meson reads

$$
C_{2 \text { pts }}(t)=\sum_{\vec{x}} \operatorname{Tr}\{S(x, 0) S(0, x)\}-2 \sum_{\vec{x}} \operatorname{Tr}\{S(x, x)\} \operatorname{Tr}\{S(0,0)\}
$$

It obtains contributions from connected and disconnected part. To approximate the disconnected part, we use color spin and volume diluted unit sources. Consider a source of type

$$
\eta_{a c}(x)=\delta_{a c} \sum_{y} \delta(x, y)
$$

where $a, c$ labels both color and spin. Solving the Dirac equation with this source gives

$$
\psi_{a}^{c}(x)=\sum_{y} D_{a b}^{-1}(x, y) \eta_{b}^{c}(y)=\sum_{y} S_{a c}(x, y)
$$

Now if we multiply by the source and sum over $c$ and spatial volume $\vec{x}$ we get

$$
\sum_{\vec{x}} \eta_{a}^{c}(\vec{x}, t) \psi_{a}^{c}(\vec{x}, t)=\sum_{\vec{x}, y} \operatorname{Tr}[S(x, y)]=\sum_{\vec{x}} \operatorname{Tr}[S(x, x)]
$$

which is the disconnected part we want to evaluate. The last equality follows from Elitzur's theorem stating that the gauge average of a gauge non-invariant object vanishes.

The largest gauge noise from this source type arises from terms $S(x, y)$, where $x$ and $y$ are nearest neighbors. This can be reduced if we consider volume diluted source of type

$$
\eta_{a, k, n}^{c}(x)=\delta_{a c} \sum_{y \in P(k, n)} \delta(x, y)
$$

where $P_{k, n}=\left\{x=\left(x_{0}, x_{1}, x_{2}, x_{3}\right) \mid x_{0}+x_{1}+x_{2}+x_{3}=k \bmod n\right\}$, i.e. the set $P_{0,2}$ is the set of even lattice sites and $P_{1,2}$ the odd ones. Now again we have

$$
\sum_{k=0}^{n-1} \sum_{x \in P_{k, n}} \eta_{a, k, n}^{c}(x) \psi_{a, k, n}^{c}(x)=\sum_{\vec{x}} \operatorname{Tr}[S(x, x)]
$$

However, the leading gauge non-invariant terms are of a distance $n$ apart and hence suppressed. Our lattice sizes $L=32 \times 16^{2}$ limits us to $n=8$. Naturally we need to perform also $n$ times more inversions. 


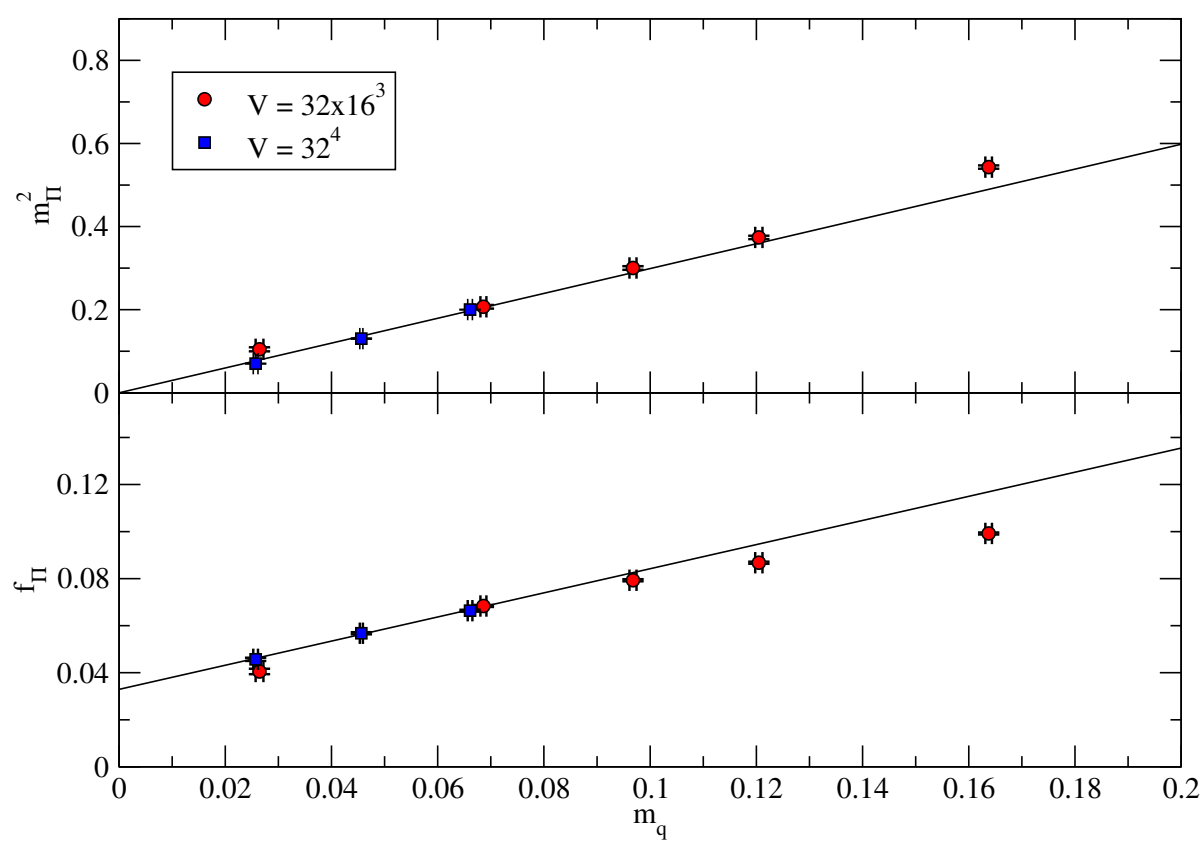

Figure 1: The chiral extrapolations of $m_{\Pi}$ and $f_{\Pi}$ as a function of the PCAC-mass $m_{q}$ in lattice units.
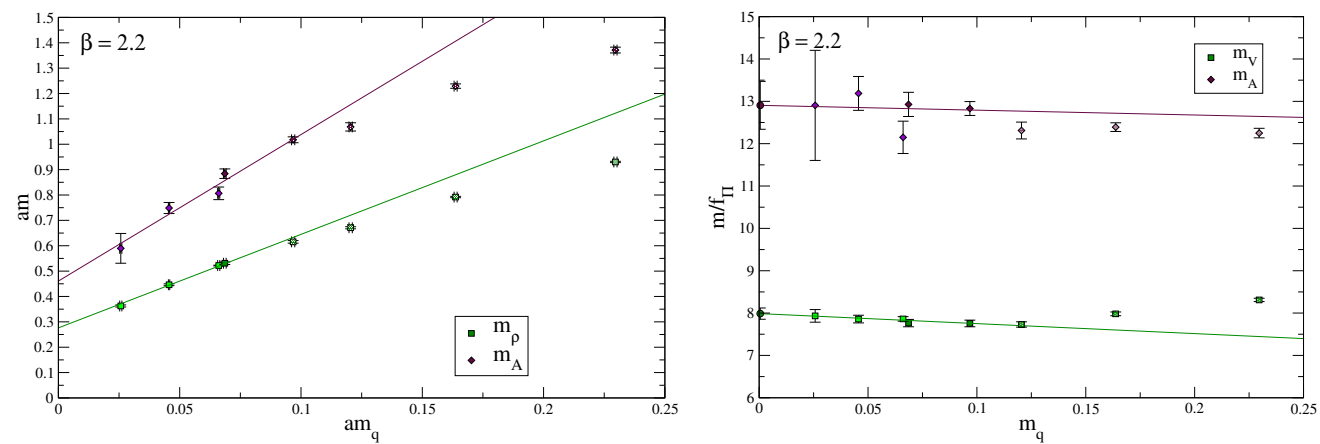

Figure 2: Left panel: Mass of vector and axial vector meson in lattice units for the finer lattice as a function of the PCAC-mass. Right panel: Mass of vector and axial vector meson as units of $f_{\Pi}$ for the finer lattice as a function of the PCAC-mass.

\section{Results}

We have performed the simulations with two different lattice spacings $\beta=2.0$ and 2.2 , and with six and seven different fermion masses respectively. We also performed some simulations varying volume to control finite volume effects. In our most chiral point in finer lattice we see finite volume effects on the smallest volume, but on larger volumes they are smaller than our statistical error.

The chiral extrapolation works qualitatively well for $m_{\Pi}$ and $f_{\Pi}$. See Fig. 1 . However, fixing 


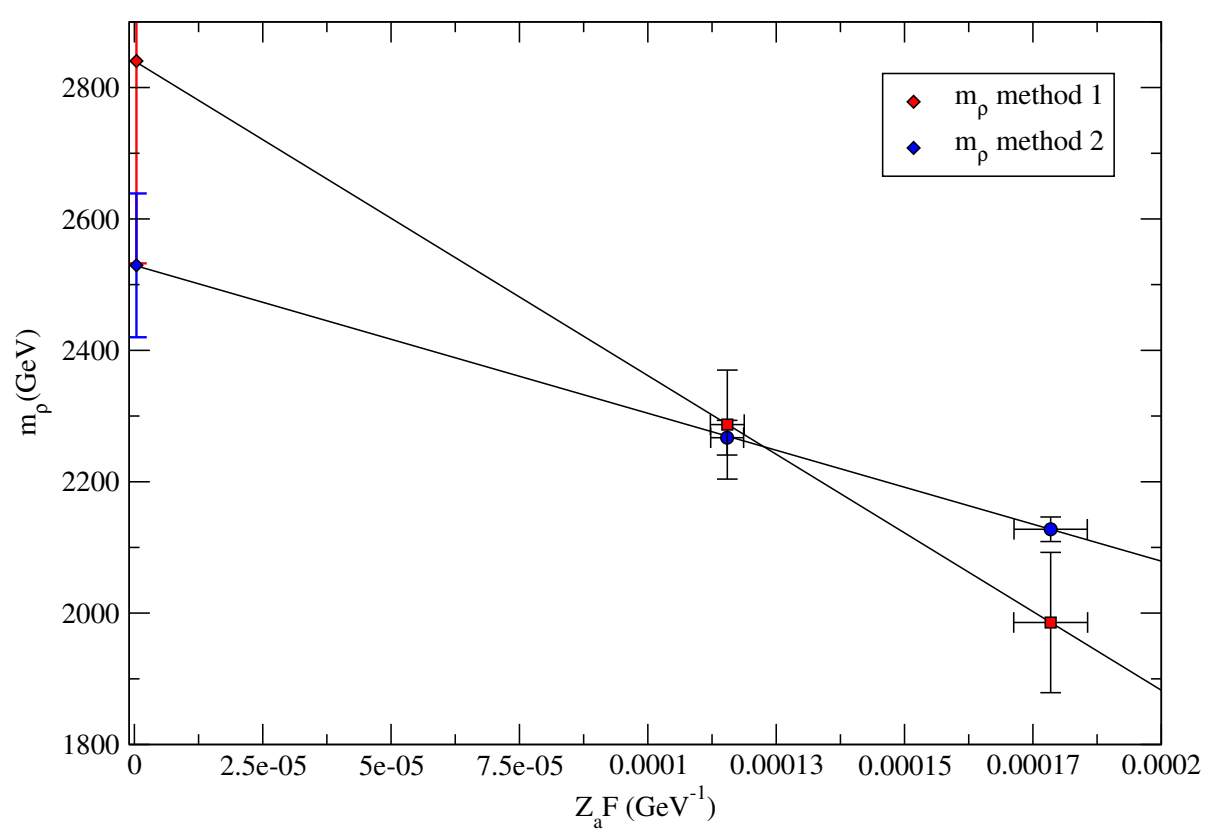

Figure 3: The continuum extrapolation of vector and axial vector meson masses as a function of lattice spacing.

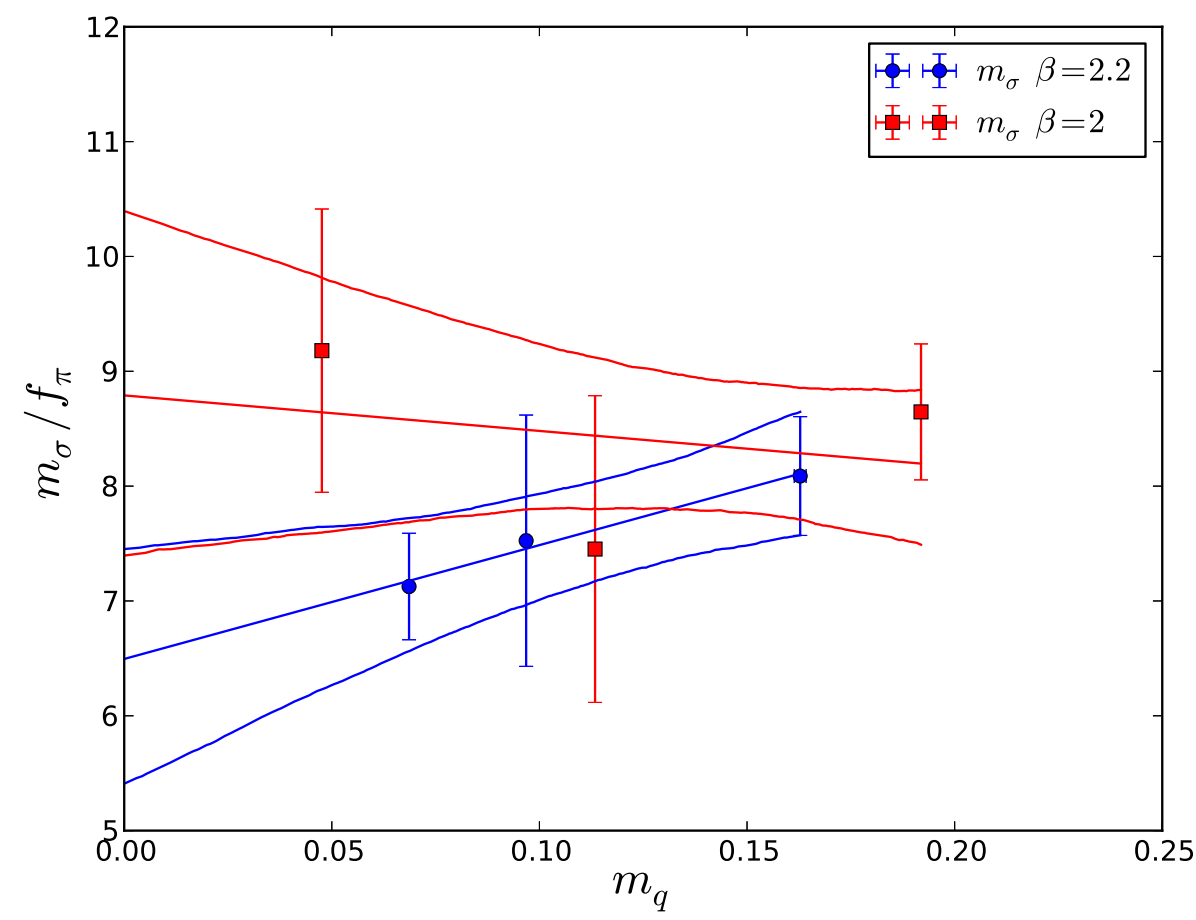

Figure 4: Scalar mass in the units of $f_{\Pi}$ as a function of the unrenormalized PCAC-mass $m_{q}$. 
the known low-energy constants $C$ and $C^{\prime}$ in the chiral perturbation theory result [12]

$$
\frac{m_{\Pi}^{2}}{m_{\mathrm{q}}}=2 B\left[1+C x \log x+D x+\mathscr{O}\left(m_{\mathrm{q}}^{2}\right)\right]
$$

and

$$
f_{\Pi}=F\left[1+C^{\prime} x \log x+D^{\prime} x+\mathscr{O}\left(m_{\mathrm{q}}^{2}\right)\right],
$$

where $B, F, D$ and $D^{\prime}$ are unknown, and $x \equiv \frac{2 B m_{q}}{16 \pi^{2} F^{2}}$., does not describe the data well.

The scale of the theory is set by requiring that $f_{\Pi} \sin \theta=246 \mathrm{GeV}$. In the extrapolation to chiral limit the scale can be set in two different ways. We can extrapolat separately $m_{\rho, A}$ and $f_{\Pi}$ to the continuum limit and take the ratio afterwards (Fig. 2), or we can take ratio of $m_{\rho, A}$ and $f_{\Pi}$ first and then extrapolate to the chiral limit (Fig. 2). The latter works better as the chiral extrapolation is almost flat, hence we use it for the central value. However, they both result to a same number within the statistical errors.

As we have only two different lattice spacings we can only perform a linear fit to the continuum extrapolated data with little control over systematic errors (see Fig. 3). Hence, we take a conservative approach, and approximate the systematic error to be the difference between the value on the finest lattice spacing and the continuum limit. Another source of systematic error is an unknown renormalization factor $Z_{a}$, which multiplies the $f_{\Pi}$. As an estimate we use its perturbative value $Z_{a}=1-0.2983 / \beta$ [13] and as an estimate of systematic error the difference of results between perturbative $Z_{a}$ and using $Z_{a}=1$. This leads to our final results:

$$
\begin{aligned}
& m_{\rho} \sin \theta=2520(100)(240)(310) \approx 2500(500) \mathrm{GeV} \\
& m_{A} \sin \theta=3300(400)(510)(340) \approx 3300(700) \mathrm{GeV} .
\end{aligned}
$$

For a reliable measurement of the scalar meson mass we need an order of 10000 configurations, and we are still in process of generating them. The connected part can be identified with isovector scalar meson and our data shows that it is heavier than the disconnected part and can be neglected. Our preliminary results are shown in Fig. 4 with two different lattice spacings and suggest that the scalar is lighter than the vector meson. Analytical predictions for the scalar mass, before electroweak and top corrections, appeared in [4] using large $\mathrm{N}$ scaling arguments and it is expected to be approximately around 1 to $1.5 \mathrm{TeV}$. These simple estimates are also supported by very recent results [14] making use of Dyson-Schwinger approximations.

\section{Summary}

We have provided phenomenological relevant information for the spectrum of an underlying gauge theory naturally unifying at the more fundamental level models of CGBH and Technicolor. The continuum extrapolated vector meson mass is $m_{\rho} \sin \theta=2.5(5) \mathrm{TeV}$. The largest errors come from the continuum extrapolation and unknown $Z_{a}$, we are in a process of improving this calculation. The preliminary results indicate that the scalar meson would be lighter than the $\rho$-meson. However, more statistics is needed to confirm this observation. Our preliminary results also hints that the lightest new state of the theory could be another scalar particle. 


\section{Acknowledgments}

This work was supported by the Danish National Research Foundation DNRF:90 grant and by a Lundbeck Foundation Fellowship grant. The computing facilities were provided by the Danish Centre for Scientific Computing.

\section{References}

[1] G. Cacciapaglia and F. Sannino, JHEP 1404, 111 (2014) [arXiv:1402.0233 [hep-ph]].

[2] T. Appelquist, P. S. Rodrigues da Silva and F. Sannino, Phys. Rev. D 60, 116007 (1999) [hep-ph/9906555].

[3] T. A. Ryttov and F. Sannino, Phys. Rev. D 78, 115010 (2008) [arXiv:0809.0713 [hep-ph]].

[4] R. Foadi, M. T. Frandsen and F. Sannino, Phys. Rev. D 87, 095001 (2013) [arXiv:1211.1083 [hep-ph]].

[5] A. Hietanen, R. Lewis, C. Pica and F. Sannino, JHEP 1407, 116 (2014) [arXiv:1404.2794 [hep-lat]].

[6] R. Arthur, V. Drach, M. Hansen, A. Hietanen, C. Pica and F. Sannino, arXiv:1412.4771 [hep-lat].

[7] R. Lewis, C. Pica and F. Sannino, Phys. Rev. D 85, 014504 (2012) [arXiv:1109.3513 [hep-ph]].

[8] A. Hietanen, R. Lewis, C. Pica and F. Sannino, arXiv:1308.4130 [hep-ph].

[9] W. Detmold, M. McCullough and A. Pochinsky, arXiv:1406.4116 [hep-lat].

[10] M. R. Buckley and E. T. Neil, Phys. Rev. D 87, 043510 (2013) [arXiv:1209.6054 [hep-ph]].

[11] P. A. Boyle, A. Juttner, C. Kelly and R. D. Kenway, JHEP 0808, 086 (2008) [arXiv:0804.1501 [hep-lat]].

[12] J. Bijnens and J. Lu, JHEP 0911, 116 (2009) [arXiv:0910.5424 [hep-ph]].

[13] L. Del Debbio, M. T. Frandsen, H. Panagopoulos and F. Sannino, JHEP 0806, 007 (2008) [arXiv:0802.0891 [hep-lat]].

[14] M. Vujinovic and R. Williams, arXiv:1411.7619 [hep-ph]. 